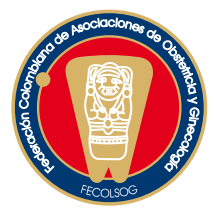

REPORTE DE CASO

\title{
MANEJO LAPAROSCÓPICO DE QUISTE GIGANTE DE OVARIO: REPORTE DE CASO
}

\author{
A case report of laparoscopic management of a \\ giant cystic ovarian mass \\ Federico Zapata-Pérez, M.D. *, Juan David Castañeda-Roldán, M.D. **, José Fernando De \\ los Ríos-Posada, M.D.**, Adriana María Arango-Martínez, M.D., M.Sc***, \\ Ricardo Anibal Mesa-Cock, M.D. **** \\ Recibido: noviembre 15/07 - Aceptado: enero 28/09
}

\section{RESUMEN}

Objetivo: revisar las consideraciones técnicas, indicaciones, riesgos y beneficios de este tipo de abordaje en masas pélvicas gigantes. Se presenta un caso en el que se realizó el abordaje laparoscópico de una masa quística ovárica de gran tamaño y su extracción a través de colpotomía posterior. La cirugía laparoscópica de los quistes gigantes de ovario surge como una alternativa a considerar en el manejo de estas pacientes.

Palabras clave: quiste gigante de ovario, laparoscopia, colpotomía.

\section{SUMMARY}

Objective: reviewing technical considerations, indications, risks and benefits of a laparoscopic approach to giant ovarian cysts. A case is presented where a giant ovarian cyst was extracted by posterior colpotomy. Laparoscopic surgery on giant pelvic mass represents an alternative for managing such patients.

* Ginecólogo, Residente Cirugía Endoscópica Ginecológica CES. Ginecólogo Clínica Las Américas. Grupo salud sexual y reproductiva, línea endoscopia ginecológica, Universidad CES. Medellín, Colombia. Correo electrónico: fedezapata69@hotmail.com

** Ginecólogo Endoscopista Clínica del Prado. Profesor Posgrado Cirugía Endoscópica Ginecológica CES. Grupo salud sexual y reproductiva, línea endoscopia ginecológica, Universidad CES. Medellín, Colombia.

*** Ginecóloga Magíster Epidemiología. Profesora Universidad CES. Grupo salud sexual y reproductiva, línea endoscopia ginecológica, Universidad CES. Medellín, Colombia.

**** Patólogo. Profesor Universidad CES, Jefe de laboratorio de patología y citología Clínica del Prado. Grupo salud sexual y reproductiva, línea endoscopia ginecológica, Universidad CES. Medellín, Colombia.
Key words: giant ovarian cyst, laparoscopy, colpotomy.

\section{INTRODUCCIÓN}

Los quistes ováricos son un problema común. En el Reino Unido son la cuarta causa de admisión hospitalaria de origen ginecológico. Se calcula que a la edad de 65 años, un 4\% de las mujeres habrán sido admitidas en un hospital por esta razón. ${ }^{1}$

Cuando los cirujanos se enfrentan a un quiste de ovario benigno de gran tamaño, la mayoría escogen la vía abierta; pocos tratan de removerlo por vía laparoscópica, principalmente debido a que se prevén dificultades técnicas, falta de entrenamiento y la posibilidad de malignidad. ${ }^{2}$

El manejo laparoscópico en masas anexiales benignas ha sido referido como seguro. ${ }^{3}$

También se ha asociado a una menor pérdida sanguínea, menores complicaciones posquirúrgicas, más corto tiempo de estadía hospitalaria y más rápida recuperación, en comparación con el manejo tradicional por laparotomía. ${ }^{4}$

Si la masa anexial es unilateral, quística, unilocular, de superficie lisa, pared delgada, sin partículas sólidas internas y sin ascitis, el riesgo de malignidad es muy bajo. El nivel normal de marcadores tumorales, especialmente del antígeno sérico CA 125, apoya mucho más la probable benignidad del tumor. En general, los tumores malignos son masas de tejido blando, con bordes irregulares, necrosis central, 
aspecto mal definido, septos gruesos y papilas en el interior. Igualmente pueden tener vegetaciones internas o externas. ${ }^{5}$

La remoción laparoscópica de masas anexiales malignas puede ser evitada con una cuidadosa selección prequirúrgica de la paciente. Si la evaluación antes del procedimiento eleva la sospecha de malignidad, se deberá optar por realizar una laparotomía. Existen herramientas adicionales, como el índice de riesgo de malignidad de Jacobs, que permiten exactitud en el diagnóstico y excluyen del manejo laparoscópico a pacientes con masas malignas. ${ }^{6}$

Algunos autores han limitado el abordaje laparoscópico a casos en los cuales la masa anexial es menor a $10 \mathrm{~cm}$. Se define quiste gigante como aquel que presenta un tamaño superior a $10 \mathrm{~cm}^{7}$

Un gran quiste de ovario impone algunos retos para el cirujano que lo aborde por laparoscopia, como son: la insuflación y la inserción del trocar, que no deben ser realizados de la manera usual, por el riesgo de ruptura y el espacio disminuido por la compresión del quiste, lo cual hace la instrumentación más difícil e incrementa el riesgo de ruptura. ${ }^{8}$

Aunque la remoción laparoscópica total es posible, existen reportes de técnicas combinadas para el abordaje de pacientes con masa anexial gigante. Se ha reportado la extracción de un quiste gigante, descomprimiéndolo por minilaparotomía y completando la ooforectomía por vía laparoscópica. ${ }^{8}$

También se ha descrito el drenaje del quiste antes del procedimiento laparoscópico con un catéter suprapúbico, seguido por cistectomía laparoscópica. ${ }^{9}$

Para la extracción de las masas gigantes luego de resecarlas por laparoscopia, es conocido el uso de bolsas endoscópicas, disponibles comercialmente, y métodos de morcelación. ${ }^{10}$ Otras opciones descritas incluyen el uso de retractores abdominales autorretenibles para minilaparotomía. ${ }^{11}$

En la literatura revisada, no se encontraron descripciones de la extracción de quistes gigantes de ovario a través de colpotomía posterior.
Este caso se presenta con el objetivo de revisar las consideraciones técnicas, indicaciones, riesgos y beneficios de este tipo de abordaje en masas pélvicas gigantes.

\section{DESCRIPCIÓN DEL CASO}

Mujer de 54 años, casada, con tres hijos, quien acudió a la Clínica del Prado de Medellín, Colombia, por un cuadro de un año de evolución de dolor abdominal progresivo, localizado en hipogastrio, intermitente, moderado, asociado a polaquiuria y empeoramiento posprandial. Refería sangrado vaginal recurrente desde hacía 8 meses, después de 7 años de menopausia. Como único antecedente quirúrgico tenía una tubectomía.

No tenía antecedentes patológicos de ningún otro tipo. En el examen físico se encontró una paciente en condiciones generales aceptables, con frecuencia cardíaca de 78 latidos por minuto, una presión arterial de 110/70 mmHg y $70 \mathrm{~kg}$ de peso.

El abdomen evidenció una masa gigante que lo ocupaba en toda su extensión, hasta el apéndice xifoides. El examen vaginal reveló un útero difícil de definir por la interferencia de la masa, y un cérvix de aspecto normal. Tenía una ecografía transvaginal y abdominal practicada un mes antes de la cirugía, que informaba la presencia de una masa quística gigante de ovario izquierdo, con medidas de 200 x 167 x $131 \mathrm{~mm}$, sin septos ni papilas en su interior, sugestiva de un cistoadenoma.

Una tomografía axial computarizada confirmó la presencia de lesión intraabdominal quística, con un tamaño mayor al reportado por ultrasonido y de probable origen anexial izquierdo; además demostró dilatación de cavidades colectoras renales y del uréter del mismo lado.

El antígeno CA 125 dio como resultado $25 \mathrm{U} / \mathrm{ml}$. Se programó para ooforectomía laparoscópica.

Bajo anestesia general, se realizó tiempo vaginal con cánula de Rubin y tiempo abdominal con aguja de Verres en el punto de Palmer, localizado en la línea medioclavicular izquierda con reborde costal, esto debido al gran tamaño del quiste. Se 
insuflaron 10 litros de $\mathrm{CO}_{2}$, insertando luego un trocar metálico de $5 \mathrm{~mm}$ y el laparoscopio. Se confirmó la presencia de la masa que ocupaba toda la cavidad abdominal, desplazando a tensión las vísceras, con un tamaño de aproximadamente $34 \mathrm{~cm}$. No había ascitis, implantes peritoneales, adherencias ni excrecencias que hicieran pensar en tumor maligno. Bajo visión directa, se insertó un trocar auxiliar de $5 \mathrm{~mm}$ en la fosa ilíaca izquierda, y a través de éste se hizo aspiración de 10 litros de líquido amarillo claro, hasta colapsar totalmente el quiste (figura 1). Luego se pasaron 2 trocares auxiliares de $5 \mathrm{~mm}$ en la fosa ilíaca derecha y área suprapúbica, y con energía bipolar a 25 watts y corte con tijera, se realizó una salpingo-ooforectomía izquierda. La evaluación del ovario contralateral, el apéndice cecal, el epiplón, el estómago y el resto de vísceras intraabdominales, no reveló patología diagnosticable por este medio. Por vía vaginal se introdujo una gasa en el fórnix posterior, y a través de la vía laparoscópica se practicó una colpotomía de 2,5 cm con 75 vatios de corriente monopolar. El anexo fue extraído sin dificultad por la incisión practicada.

Figura 1. Quiste gigante después de aspiración laparoscópica.

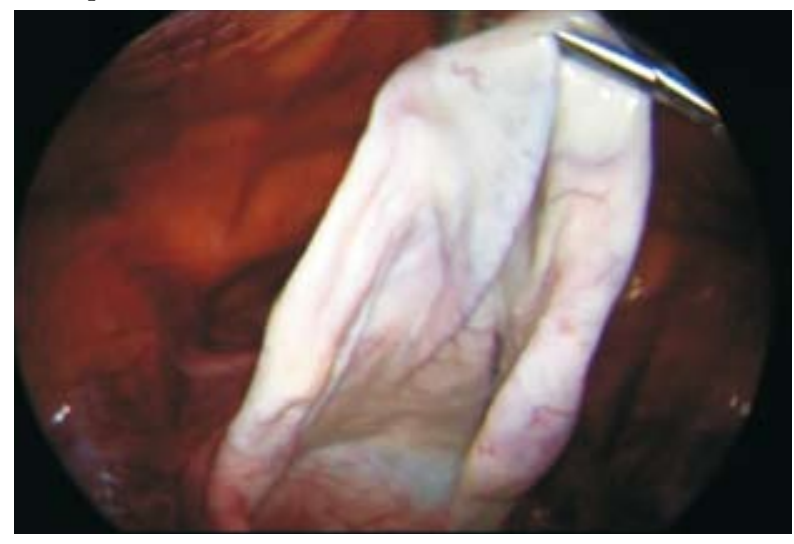

Por vía vaginal se cerró la colpotomía con una sutura continua de catgut cromado 2-0, y a través del abdomen se verificó la hemostasia en la zona de resección. No hubo complicaciones y la paciente fue dada de alta 2 horas después de la intervención.
En la revisión posoperatoria, ocho días más tarde, la paciente refirió mejoría total de sus síntomas y dijo sentirse satisfecha con su tratamiento. La anatomía patológica reportó una lesión quística benigna tapizada por epitelio cilíndrico compatible con cistadenoma seroso (figura 2).

Figura 2. Microscopía de la lesión.

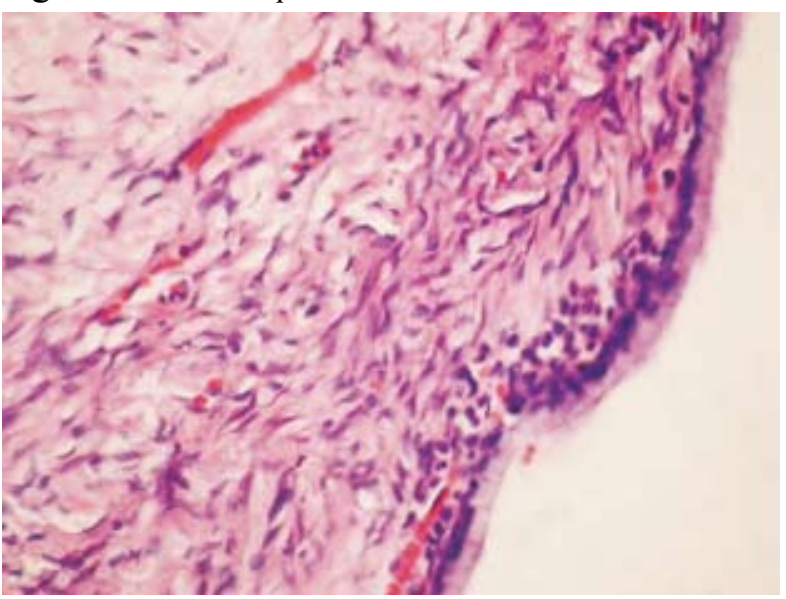

\section{DISCUSIÓN}

Cuando se opera un quiste de ovario con sospecha de malignidad, debe evitarse al máximo el escape del líquido a la cavidad peritoneal. Los implantes del tumor en la pared abdominal ocurren en el 1,1\% de las pacientes. ${ }^{12}$

Se ha sugerido que los quistes gigantes pueden ser removidos por vía laparoscópica si cumplen varias condiciones, a saber:

1. Evaluación prequirúrgica que indique baja probabilidad de malignidad.

2. Remoción intacta del quiste ovárico.

3. Cuidadosa aspiración del quiste y sus contenidos.

4. Buen lavado peritoneal, si hay ruptura del quiste. ${ }^{13}$

La técnica recomendada incluye la utilización del punto de Palmer para ingresar a la cavidad abdominal. Después de realizar la insuflación, se debe valorar la cavidad abdominal entera, inspeccionando el hígado, goteras parietocólicas, superficies peritoneales, estómago, intestino, la cápsula del quiste y el ovario contralateral. Si se considera necesario, se deben tomar muestras de líquido peritoneal. La 
punción con una aguja laparoscópica y cánula de succión permiten aspirar y drenar el quiste, evitando el escape del líquido. Aunque algunos autores consideran que el escape de células a la cavidad peritoneal, cuando se presenta una ruptura intraoperatoria, empeora el devenir en casos de malignidad inadvertida, otros trabajos han sugerido que esto no cambia el pronóstico. Estos estudios han determinado que los únicos factores que influencian de forma importante la recaída del tumor son: el grado del tumor, la presencia de adherencias densas o la presencia de ascitis de gran volumen, y que no hay diferencia en la tasa de supervivencia a 5 años entre los pacientes a los que se les hace remoción intacta del quiste, comparada con aquellos con ruptura del mismo en la cirugía. ${ }^{14}$

La adecuada evaluación prequirúrgica con los índices de riesgo y con Doppler en masas anexiales, puede aumentar la sensibilidad para la detección un poco más precisa de la probable naturaleza maligna en este tipo de tumores. ${ }^{15}$

Un informe reciente valoró el abordaje laparoscópico para quistes gigantes benignos de ovario en 33 pacientes, encontrando una tasa de éxito del 93\%. Dos pacientes requirieron laparotomía por adherencias. El 70\% de las pacientes fueron dadas de alta el mismo día de la cirugía. No hubo complicaciones intra ni posoperatorias. ${ }^{16}$

\section{REFERENCIAS}

1. Garcetti GG, Ciavattini A, Tiriduzzi M. Combined ultrasonographically guided drainage and laparoscopic excision of large endometriomas. A pilot study. Gynecol Obstet Invest 1998;45:266-8.

2. Goh SM, Yam J, Loh SF, Wong A. Minimal access approach to the management of large ovarian cysts. Surg Endosc 2007;21:80-3.

3. Yuen PM, Yu KM, Yip SK. A randomized prospective study of laparoscopy and laparotomy in the management of benign ovarian masses. Am J Obstet Gynecol 1997;177:109-14.
4. Mais V, Ajossa S, Piras B, Maroingiu D, Guerriero S, Melis GB. Treatment of nonendometriotic benign adnexal cysts: a randomized comparison of laparoscopy and laparotomy. Obstet Gynecol 1995;86:770-4.

5. Knudsen UB, Tabor A, Mosgaard B, Andersen ES, Kjer JJ, Hahn-Petersen S, et al. Management of ovarian cyst. Acta Obstet Gynecol Scand 2004;83:1012-21.

6. Jacobs I, Oram D, Fairbanks J, Turner J, Frost C, Grudzinkas JG. A risk of malignancy index incorporating CA 125, ultrasound and menopausal status for the accurate preoperative diagnosis of ovarian cancer. Br J Obstet Gynaecol 1990;97:922-9.

7. Salem HA. Laparoscopic excision of large ovarian cysts. J Obstet Gynaecol Res 2002;28:290-4.

8. Canarelli JP, Plancq MC, Bob’Oyono JM, Ricard J. Voluminous ovarian cyst children. Therapeutic strategy. J Chir (Paris) 1993;130:525-8.

9. Dolan MS, Boulanger SC, Salameh JR. Laparoscopic management of giant ovarian. JSLS 2006;10:254-6.

10. Eltabbakh GH, Kaiser JR. Laparoscopic management of a large ovarian cyst in an adolescent. A case report. J Reprod Med 2000;45:231-4.

11. Sunoo CS. Laparoscopic removal of a large adnexal mass. Obstet Gynecol 2004;103:1087-9.

12. Rhode JM, Advincula AP, Reynolds RK, Bruke WM. A minimally invasive technique for management of the large adnexal mass. Journal of Minimally Invasive Gynecology 2006;13:476-9.

13. Childers JM, Aqua KA, Surwit EA, Hallum AV, Hatch KD. Abdominal-wall tumor implantation after laparoscopy for malignant conditions. Obstet Gynecol 1994;84:765-9.

14. Vergote I, De Brabanter J, Fyles A, Bertelsen K, Einhorn N, Sevelda P, et al. Prognostic importance of degree of differentation and cyst rupture in stage I invasive epitelial ovarian carcinoma. Lancet 2001;357:176-82.

15. Loh SF, Yan YL, Huang HF, Yeo GSH. Efficacy of colour Doppler imaging in the pre-operative diagnosis of ovarian malignancy. Free paper FIGO Copenhagen; 1997.

16. Eltabbakh GH, Charboneau AM, Eltabbakh NG. Laparoscopic sugery for large benign ovarian cysts. Gynecol Oncol 2008;108:72-6. 